\title{
Prevalence of urinary tract infections and current scenario of antibiotic susceptibility pattern of bacteria causing UTI
}

\author{
Pritam Pardeshi \\ Consultant Microbiologist, Dept. of Microbiology, Bhatia Hospital, Mumbai, Maharashtra, India
}

*Corresponding Author:

Email: dr.pritampardeshi@gmail.com

Received: $8^{\text {th }}$ July, 2018

Accepted: $25^{\text {th }}$ July, 2018

\begin{abstract}
Introduction: Urinary tract infection (UTI) is one of the most prevalent diseases affecting people of all age groups i.e. from neonate to geriatric age group. Widespread use of antibiotics has led to the emergence of resistant microorganisms. As the antibiogram of the microorganisms are frequently changing hence the present study was done to analyze the recent antibiotic sensitivity pattern of uropathogens in urinary tract infection.

Materials and Methods: In present study is a retrospective analysis of culture results of urine samples, was conducted at Microbiology department of tertiary health care hospital in Mumbai. The age \& sex of patients, the organism isolated and also the antimicrobial susceptibility profiles were collected from the laboratory registers using a standard data collection form.

Results: The overall prevalence of UTI was $33.54 \%$ of which $66.78 \%$ were females and $33.22 \%$ were from males. High prevalence was observed in females as compared to males (2:1). Though the overall prevalence was high in old aged (>45 years) patients, in females high prevalence was seen among middle-aged (31 to 45 years) patients and in male high prevalence was seen among old age (>45 years) patients. From total 584 uropathogens, E.coli $(53.77 \%)$ was the commonest isolate causing UTI followed by Klebsiella pneumoniae (27.40\%). The most effective antimicrobial agents in our study were Meropenem, gentamicin, Nitrofurantoin and Cotrimoxazole whereas higher resistance was observed among Fluoroquinolones, Amoxicillin and third generation Cephalosporins, these are the drugs which are commonly given emperically for UTI.

Conclusion: As drug resistance among bacterial pathogens is vary with time to time regular surveillance and monitoring is necessary for giving updated information to physician for most effective empirical treatment of UTIs.
\end{abstract}

Keywords: Urinary tract infection, Prevalence, Antibiotics resistance, Uropathogenes.

\section{Introduction}

Urinary tract infection (UTI) is one of the most prevalent disease affecting people from all age groups including neonate and geriatric age groups. Every year about 150 million people are being diagnosed with urinary tract infection worldwide. Each and every woman has a lifetime risk of developing UTI is $60 \%$; by contrast, men have a lifetime risk of only $13 \%{ }^{1}$

Clinically, UTI is divided into two categories, uncomplicated and complicated. Uncomplicated UTI mostly affects healthy individuals with no structural or neurological urinary tract abnormalities; which includes cystitis and pyelonephritis. Complicated UTI is due to the factors that compromise the urinary tract, which include urinary obstruction, neurological disease causing urinary retention, renal failure, renal transplantation, pregnancy and the presence of foreign bodies such as calculi, indwelling catheters or other drainage devices. ${ }^{2}$

Females are more susceptible to UTI as compared to males due to the short length of urethra, absence of prostatic secretion, pregnancy and easy contamination of the tract with faecal flora. ${ }^{3}$

E. coli is most common organism causing UTI which accounts for up to $90 \%$ of cases. P. mirabilis, Klebsiella species, P. aeruginosa and Enterobacter species are less frequent offenders. Gram-positive organisms are less common which includes Group B
Streptococcus, S. aureus, S. saprophyticus and $S$. haemolyticus. ${ }^{4}$

Currently UTI is mostly managed empirically without urine culture or susceptibility testing this may lead to the frequent misuse of antibiotics. The antimicrobial susceptibility data of UTI-causing microorganisms is variable it changes from time to time and from place to place. Most commonly UTIs are treated empirically; in that case the criteria for the selection of antimicrobial agents should be determined on the basis of the most likely pathogen and its expected resistance pattern in that geographic area. Hence, the periodic monitoring of etiologic agents of UTI and their resistance pattern in the community is very essential.

The aim of the study is to determine the age wise and sex wise prevalence of UTI and to analyze the recent sensitivity pattern of Uropathogens in the management of UTI which may help and guide the Medical Practitioners to carry out empirical treatment.

\section{Materials and Methods}

Study Design: The Present study is a retrospective study on UTI in which analysis of urinary culture results are done. This study, conducted at the Microbiology department of a tertiary health care hospital in Mumbai. The age, sex, the organism isolated and the antimicrobial susceptibility profiles were 
collected from the records. The data were entered into Excel for analysis and statistical analysis was done.

Culture and Identification: Urine specimens were collected in sterile wide mouth glass container as per the standard operating procedures. Urine samples were plated using calibrated wire loops $(0.001 \mathrm{ml})$ on Cystine Lactose Electrolyte-Deficient (CLED) medium, MacConkey agar and blood agar and then incubated aerobically at $37{ }^{\circ} \mathrm{C}$ for $24 \mathrm{~h}$. From positive cultures, isolates were identified according to the standard operating procedures. An isolate was considered significant if urine cultures having colony count $\geq 10^{5}$ $\mathrm{CFU} / \mathrm{ml}^{5}$

Antimicrobial Susceptibility Tests: Antimicrobial susceptibility tests were done as per Kirby-Bauer disk diffusion method. ${ }^{6}$ The antimicrobial agents tested were: Nitrofurantoin $(300 \mu \mathrm{g})$, Azithromycin $(15 \mu \mathrm{g})$, Gentamicin $(10 \mu \mathrm{g})$, Ciprofloxacin $(5 \mu \mathrm{g})$, Norfloxacin (10 $\mu \mathrm{g})$, Trimethoprim-Sulphamethoxazole (cotrimoxazole) (1.25-23.75 $\mu \mathrm{g})$, Ceftriaxone $(30 \mu \mathrm{g})$ and Amoxycillin $(10 \mu \mathrm{g})$, Amoxycillin-Clavulinic Acid, Tetracycline $(30 \mu \mathrm{g})$, Cefepime $(30 \mu \mathrm{g})$, Meropenem (10 $\mu \mathrm{g})$. Resistance data were interpreted according to Clinical laboratory Standards Institute (CLSI). ${ }^{7}$

Approval taken from medical Bhatia hospital Medical Research Society.

\section{Results}

A total of 1741 cases of symptomatic UTI were studied in one year, among these, no infectious etiology was found in $1157(66.46 \%)$.

The overall prevalence of UTI was found to be $33.54 \%$ (584/1741). Total 584 urine samples showed the significant bacterial growth, 194 (33.22\%) samples from males and $390(66.78 \%)$ from female patients (Table 1). In females high prevalence was seen among middle-aged ( 31 to 45 years) patients and in male high prevalence was seen among old age ( $>45$ years) patients (Table 1).

There was not any significant seasonal variation in the prevalence of infection. However, maximum cases occurred in the hot-humid summer, in the months of April to September (Fig. 1).

From total 584 uropathogens, E.coli $(53.77 \%)$ was the commonest isolate causing UTI. The second most prevalent isolate was Klebsiella pneumoniae (27.40\%) followed by Pseudomonas aeruginosa (8.56\%), Proteus spp. (4.79\%), Enterobacter spp. (1.71\%), Staphylococcus aureus (1.54\%) (Fig. 2).

Fig. 3 is representative of overall antibiotic sensitivity pattern of urinary bacterial isolates. Gentamicin, Nitrofurantoin and Sulphamethoxazole/Trimethoprim (Co-Trimoxazole were found to be agents with high sensitivity of various uro-pathogens. Other antibiotics with higher sensitivity were Tetracycline \& Meropenem. Overall susceptibility of organisms showed considerable resistance to Ampicillin, Fluoroquinolones and Ceftriaxone with low susceptibilities ranging from 20-30\%. However, Gentamicin (59.58\%), Nitrofurantoin $(52.91 \%)$ and Sulphamethoxazole/Trimethoprim (Co-Trimoxazole) $(49.49 \%)$ showed relatively good susceptibility, thus providing a better option for treatment.

Percentage sensitivity of organism to antimicrobial agents is given in Table 2.

Table 1: Age and sex prevalence of UTI

\begin{tabular}{|l|c|c|c|c|c|c|}
\hline \multirow{2}{*}{ Age } & \multicolumn{2}{c|}{ Male } & \multicolumn{2}{c|}{ Female } & \multicolumn{2}{c|}{ Total } \\
\cline { 2 - 7 } & No. & $\mathbf{\%}$ & No. & \% & No. & \% \\
\hline$<$ 18 years & 21 & 3.59 & 7 & 1.20 & 28 & 4.79 \\
\hline 18 to 30 years & 12 & 2.05 & 57 & 9.76 & 69 & 11.81 \\
\hline 31 to 45 years & 16 & 2.74 & 184 & 31.51 & 200 & 34.24 \\
\hline$>$ 45 years & 145 & 24.83 & 142 & 24.32 & 287 & 49.14 \\
\hline Total & 194 & 33.21 & 390 & 66.79 & 584 & 100.00 \\
\hline
\end{tabular}

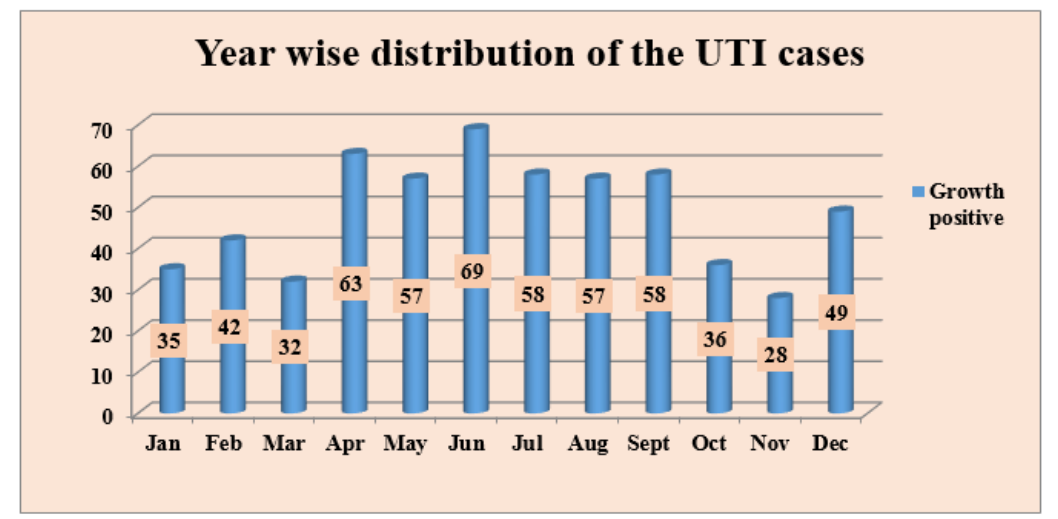

Fig. 1: Year wise distribution of the UTI cases 


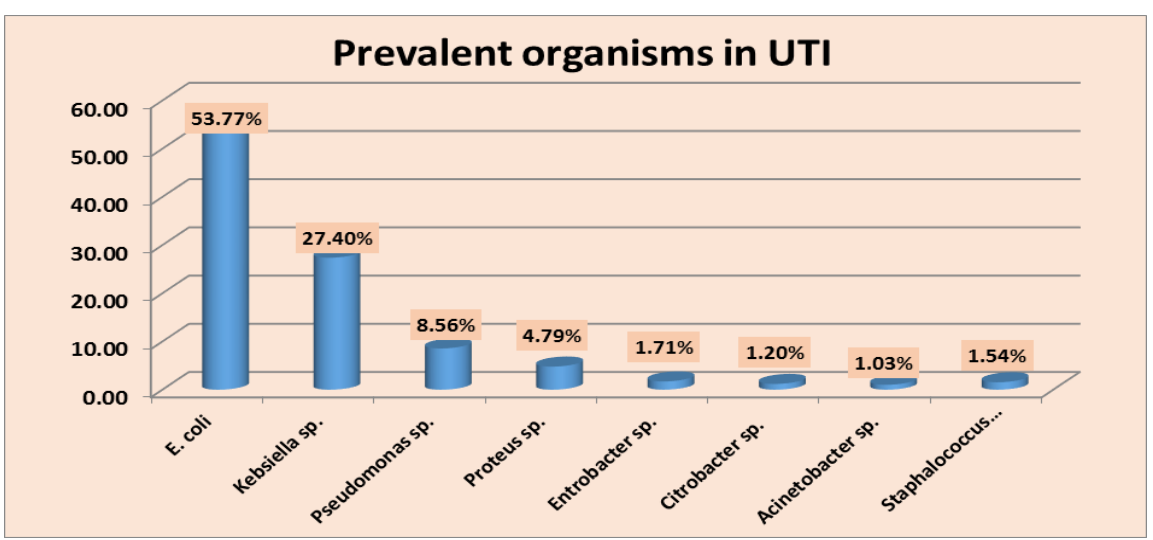

Fig. 2: Prevalent organisms in UTI

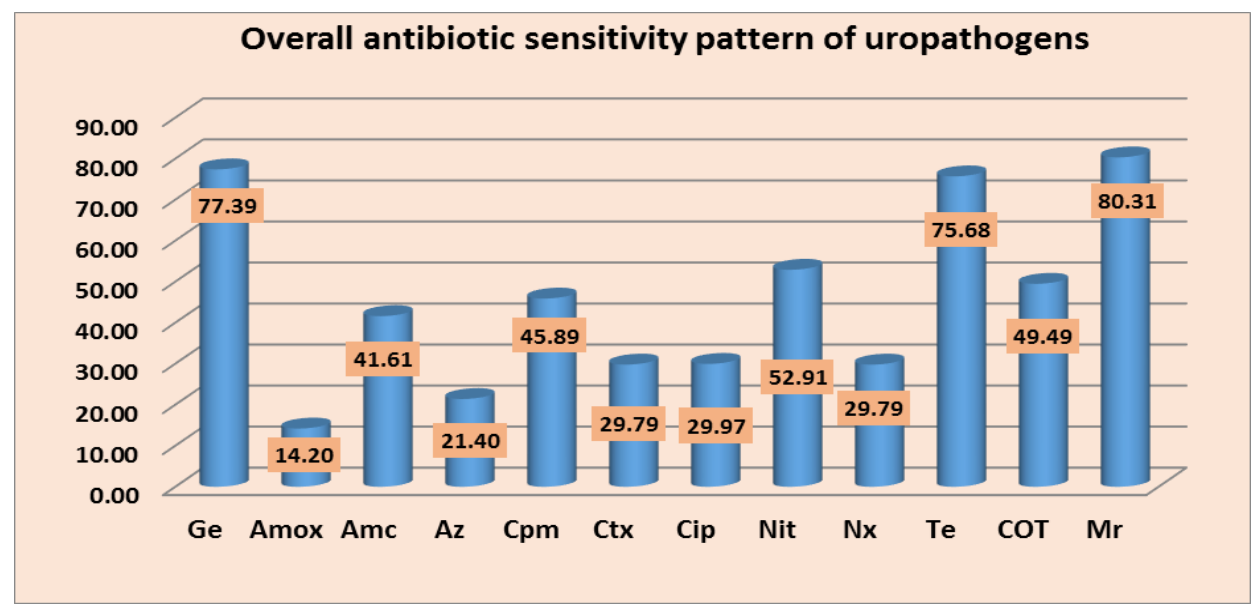

Fig. 3: Overall antibiotic sensitivity pattern of uropathogens

$\mathrm{Ge}=$ Gentamicin; Amox = Amoxycillin; Amc = Amoxycillin- Clavulinic Acid; Az= Azithromycin ; $\mathrm{Cpm}=$ Cefepime $; \mathrm{Ctx}=$ Ceftriaxone Cip = Ciprofloxacin; Nit = Nitrofurantoin; $\mathrm{Nx}=$ Norfloxacin; $\mathrm{Te}=$ Tetracycline; $\mathrm{COT}=$ Sulphamethoxazole/Trimethoprim $($ Co-Trimoxazole $) ; \mathrm{Mr}=$ Meropenem

Table 2: Percentage distribution of drug sensitivity of uropathogens

\begin{tabular}{|c|c|c|c|c|c|c|c|c|c|c|c|c|c|c|}
\hline \multirow[t]{3}{*}{ Antibiotics } & \multirow{2}{*}{\multicolumn{2}{|c|}{ E. coli (314) }} & \multirow{2}{*}{\multicolumn{2}{|c|}{$\begin{array}{c}\text { Klebsiella } \\
\text { Pneu. (160) }\end{array}$}} & \multirow{2}{*}{\multicolumn{2}{|c|}{$\begin{array}{c}\text { Pseudomo- } \\
\text { nas a. (50) }\end{array}$}} & \multirow{2}{*}{\multicolumn{2}{|c|}{$\begin{array}{l}\text { Proteus } \\
\text { Sp. (28) }\end{array}$}} & \multirow{2}{*}{\multicolumn{2}{|c|}{$\begin{array}{l}\text { Entrobac- } \\
\text { ter sp. (10) }\end{array}$}} & \multirow{2}{*}{\multicolumn{2}{|c|}{$\begin{array}{l}\text { Acitenoba- } \\
\text { cter sp. (6) }\end{array}$}} & \multirow{2}{*}{\multicolumn{2}{|c|}{$\frac{\text { Staph. }}{\text { Aureus (9) }}$}} \\
\hline & & & & & & & & & & & & & & \\
\hline & No & $\% \mathrm{~S}$ & No & $\% \mathrm{~S}$ & No & $\% \mathrm{~S}$ & No & $\% \mathrm{~S}$ & No & $\% \mathrm{~S}$ & No & $\% \mathrm{~S}$ & No & $\% \mathrm{~S}$ \\
\hline Ge & 285 & 90.76 & 109 & 68.13 & 16 & 32.00 & 27 & 96.43 & 6 & 60.00 & 5 & 83.33 & 4 & 44.44 \\
\hline Amox & 56 & 17.83 & 12 & 7.50 & 0 & 0.00 & 13 & 46.43 & 0 & 0.00 & 0 & 0.00 & 2 & 22.22 \\
\hline Amc & 162 & 51.59 & 59 & 36.88 & 0 & 0.00 & 15 & 53.57 & 1 & 10.00 & 4 & 66.67 & 2 & 22.22 \\
\hline $\mathbf{A z}$ & 68 & 21.66 & 37 & 23.13 & 3 & 6.00 & 9 & 32.14 & 2 & 20.00 & 5 & 83.33 & 1 & 11.11 \\
\hline Cpm & 135 & 42.99 & 84 & 52.50 & 17 & 34.00 & 20 & 71.43 & 6 & 60.00 & 4 & 66.67 & 2 & 22.22 \\
\hline Ctx & 102 & 32.48 & 42 & 26.25 & 7 & 14.00 & 17 & 60.71 & 1 & 10.00 & 4 & 66.67 & 1 & 11.11 \\
\hline Cip & 94 & 29.94 & 48 & 30.00 & 9 & 18.00 & 13 & 46.43 & 5 & 50.00 & 4 & 66.67 & 2 & 22.22 \\
\hline Nit & 250 & \begin{tabular}{|l|}
79.62 \\
\end{tabular} & 51 & 31.88 & 0 & 0.00 & 0 & 0.00 & 2 & 20.00 & 6 & 100.00 & 0 & 0.00 \\
\hline $\mathbf{N x}$ & 89 & 28.34 & 50 & 31.25 & 9 & 18.00 & 14 & 50.00 & 5 & 50.00 & 4 & 66.67 & 3 & 33.33 \\
\hline Te & 119 & 37.90 & 48 & 30.00 & 0 & 0.00 & 0 & 0.00 & 5 & 50.00 & 5 & 83.33 & 4 & 44.44 \\
\hline Cot & 169 & 53.82 & 86 & 53.75 & 0 & 0.00 & 21 & 75.00 & 5 & 50.00 & 5 & 83.33 & 3 & 33.33 \\
\hline $\mathrm{Mr}$ & 302 & 96.18 & 113 & 70.63 & 13 & 26.00 & 27 & 96.43 & 6 & 60.00 & 6 & 100.00 & 2 & 22.22 \\
\hline
\end{tabular}

$\mathrm{Ge}=$ Gentamicin; Amox = Amoxycillin; Amc = Amoxycillin- Clavulinic Acid; Az= Azithromycin; Cpm= Cefepime; $\mathrm{Ctx}=$ Ceftriaxone; $\mathrm{Cip}=$ Ciprofloxacin; Nit $=$ Nitrofurantoin; Nx=Norfloxacin; Te= Tetracycline; COT=Sulphamethoxazole/Trimethoprim (Co-Trimoxazole); $\mathrm{Mr}=$ Meropenem

\section{Discussion}

UTI is a one of the most common diseases diagnosed worldwide. Availability of new antimicrobials has improved the management of UTIs.
However, the management of UTI infections has been jeopardized by increase in emergence of antimicrobial drug resistance. 
The prevalence of UTI was found to be $33.54 \%$ in this study correlating to the prevalence rate of UTI with other studies from India done by M. Dash et $\mathrm{al}^{8}$ and M. Mehta et $\mathrm{al}^{9}$ showing prevalence of $34.5 \%$ and $36.68 \%$ respectively. However the higher prevalence was seen in a study by Devanand et al $(53.82 \%) .{ }^{10}$

Our study showed a high prevalence of UTI in females $(66.78 \%)$ than in males $(33.22 \%)$ which correlate with findings from other studies which revealed that the frequency of UTI is greater in females as compared to males. ${ }^{10-14}$ The reason behind this high prevalence of UTI in females is due to close proximity of the urethral meatus to the anus, shorter urethra, sexual intercourse, incontinence, and bad toilet. ${ }^{10}$ However, higher incidence was observed in middle age females and old age males may be due to prostate disease in males are responsible for the increase in the incidence of UTI above 45 years. Similar observation being reported by Smita $\mathrm{S}$ et al ${ }^{11}$ and Devanand et al. ${ }^{10}$

In the present study the overall occurrence of UTI recorded was highest among the elderly ( $\geq 48$ years, $49.5 \%$ ) compared to young and middle age patients (18 to 30 years, $12.5 \%$; 31 to $45,33.5 \%) .{ }^{10-11}$

In present study, the Gram negative bacilli contribute to $98.50 \%$ of the total bacterial isolates while Gram positive cocci constituted $1.50 \%$. Escherichia coli $(53.77 \%)$ was found to be the most common gram negative bacteria causing UTI. This result is consistent with reports from other studies by Devanand et al, ${ }^{10}$ Smita et al, ${ }^{11}$ Agbawa et al ${ }^{12}$ Mulugeta et al, ${ }^{13}$ Asrat Aglu et al, ${ }^{14}$ Arghya Das et al, ${ }^{15}$ Ahmed Naeem et al ${ }^{16}$ where $42.58 \%, 61.84 \%, 63.3 \%, 60.29 \%, 53.69 \%$, $34.1 \%$ cultures grew E. coli respectively; and it was most frequent pathogen causing UTI in all these studies. Other isolated bacteria from UTI cases in this study were $K$. pneumonia $(27.4 \%), P$. aeruginosa (8.56\%), Proteus spp. (4.79\%), Enterobacter spp. $(1.71 \%)$ and $S$. aureus $(1.54 \%)$. These results also correlate with other studies in which Klebsiella spp. was reported as the second most frequently isolated organism in UTI. ${ }^{10,13,16}$ Higher incidence of gram negative bacteria, related to Enterobacteriaceae, in causing UTI has many factors which are responsible for their attachment to the uroepithelium such as they are able to colonize in the urogenital mucosa with adhesins, pili, fimbriae, and $\mathrm{P}-1$ blood group phenotype receptor. ${ }^{10}$

The most effective antimicrobial agents in said study were Meropenem, Gentamicin, Nitrofurantoin and Cotrimoxazole showing percentage susceptibility $80.31 \%, 59.58 \%, 52.91 \%$ and $49.49 \%$ respectively (Fig. 3). Higher resistance was observed among Fluoroquinolones, Amoxicillin and third generation Cephalosporins showing percentage susceptibility of $29.79 \%, 14.20 \%$ and $29.79 \%$ respectively. Study by Ahmed Naeem et al ${ }^{16}$ has reported highest sensitivity to Gentamicin (92.3\%) followed by Imipenem (90.2\%) and less sensitivity was shown to Cefotaxime. Arghya
Das et $\mathrm{al}^{15}$ in his study observed highest resistance to Ampicillin, fluroquinolones and ceftriaxone whereas Gentamicin and Nitrofurantoin were the antibiotics to which organisms were most sensitive.

In this study, it was observed that the susceptibility of the isolates to the tested antibiotics differed with the species. Of the E. coli isolates $(53.77 \%)$ showed resistance to the commonly used antibiotics. The highest sensitivity was shown by Gentamicin $(90.76 \%)$, Meropenum (96.8\%) followed by Nitrofuration (79.62\%). High resistance to antibiotics shown by Klebsiella. Klebsiella showed highest sensitivity to Gentamicin is $68.13 \%$ and Meropenum is $70.63 \%$.

In present study overall sensitivity is observed to be very low. Antimicrobial resistance is a very huge problem throughout the world. Periodic assessment of in vitro susceptibility pattern of urinary pathogens serves as a guide for antibiotic therapy, as these organisms exhibit resistance to many first-line drugs used for UTI infection. In order to prevent resistance to antibiotics, appropriate therapy as per bacterial sensitivity pattern needs to be initiated.

This retrospective study is based on the results of routine microbiological tests carried out in 2017. Due to the nature of the retrospective analysis, we couldn't trace patient's clinical settings. Thus the study did not consider the features such as inpatient and outpatients, catheterized and non-catheterized patients.

\section{Conclusion}

As drug resistance among bacterial pathogens is changing with time and place, regular surveillance and monitoring is very essential to provide physicians updated information on most effective empirical treatment of UTIs. Empirical antibiotic choice in treatment of UTI should be based on the knowledge of local prevalence of causative micro-organisms and their antibiogram and not on universal guidelines.

\section{Acknowledgements}

I am very thankful to Bhatia hospital, Microbiology department and Bhatia hospital Medical Research Society for allowing me to conduct the study.

\section{References}

1. R Nalini, J Ezhil Ramya. Recent Sensitivity Pattern of Escherichia Coli in Urinary Tract Infection. RRJMB. 2014;3(3).

2. Ana L. Flores-Mireles, Jennifer N. Walker. Urinary tract infections: epidemiology, mechanisms of infection and treatment options. Nat Rev Microbiol. 2015;13(5):269284.

3. Singh Randhir K, Dewasy Bijoylakshmi. Prevalence of antibiotic sensitivity pattern of uropathogens in patients of different age-groups from western region of Nepal. International Journal of Medical Research \& Health Sciences. 2016;5(9):1-7.

4. KY Loh, N Sivalingam. Urinary Tract Infections in Pregnancy. Malaysian Family Physician 2007; Volume 2, Number 2. 
5. Cheesbrough, M. (2005). District Laboratory Practice in Tropical Countries Part 1, second edition. Cambridge University Press, Cambridge UK, 105-115.

6. Bauer AW, Kirby WMM, Sherris JC, Turck M. Antibiotic susceptibility testing by standard single disc method. Am J Clin Pathol. 1966;45:493-496.

7. Clinical and Laboratory Standards Institute. Performance standards for antimicrobial susceptibility testing; $27^{\text {th }}$ informational supplement. Wayne, PA: Clinical and Laboratory Standards Institute; 2017.

8. M. Dash, S. Padhi, I. Mohanty, P. Panda, and B. Parida. Antimicrobial resistance in pathogens causing urinary tract infections in a rural community of Odisha, India. Journal of Family and community medicine. 2013;20(1):20-26.

9. M. Mehta, S. Bhardwaj, and J. Sharma. Screening of urinary isolates for the prevalence and antimicrobial susceptibility of Enterobacteria other than Escherichia coli. International Journal of Life Science and Pharma Research. 2013;3(1):100-104.

10. Devanand Prakash and Ramchandra Sahai Saxena. Distribution and Antimicrobial Susceptibility Pattern of Bacterial Pathogens Causing Urinary Tract Infection in Urban Community of Meerut City, India. ISRN Microbiology. Volume 2013, Article ID 749629, 13 pages.

11. Sood S, Gupta R. Antibiotic resistance pattern of community acquired uropathogens at a tertiary care hospital in Jaipur, Rajasthan. Indian J Community Med. 2012;37:39-44.
12. Agbagwa O. E, Ifeanacho Emeka J. U. The Prevalence of UTI Pathogens in Urine Specimen Obtained from a Hospital in Rivers State Nigeria. Journal of Microbiology Research. 2015;5(5):143-148.

13. Mulugeta Kibret, Bayeh Abera. Prevalence and antibiogram of bacterial isolates from urinary tract infections at Dessie Health Research Laboratory, Ethiopia. Asian Pac J Trop Biomed. 2014;4(2):164-168.

14. Asrat Agalu Abejew, Ayele A Denboba. Prevalence and antibiotic resistance pattern of urinary tract bacterial infections in Dessie area, North-East Ethiopia. BMC Research Notes. 20147:687.

15. Arghya Das and Tuhina Banerjee, Prevalence of Urinary Tract Infections and Susceptibily Pattern of Uropathogens in Women of Reproductive age Group from North India. Journal of Advances in Medicine. 2015;4(1-2).

16. Ahmad Naeem Sajed, Uzma Batool. Prevalence of urinary tract infections and their antibiotic sensitivity in tertiary care hospital Lahore. IOSR Journal of Dental and Medical Sciences (IOSR-JDMS). 2014;13(12)Ver. II,5761.

How to cite this article: Pardeshi $P$. Prevalence of urinary tract infections and current scenario of antibiotic susceptibility pattern of bacteria causing UTI. Indian J Microbiol Res. 2018;5(3):334-338. 